\title{
Coulisses
}

Revue de théâtre

14 | Printemps 1996

Varia

\section{Lecture critique du spectacle Équations}

\section{Danielle Vingler}

\section{OpenEdition}

Journals

Édition électronique

URL : http://journals.openedition.org/coulisses/4715

DOI : $10.4000 /$ coulisses.4715

ISSN : 2546-9460

\section{Éditeur}

Presses universitaires de Franche-Comté

\section{Édition imprimée}

Date de publication : 1 mai 1996

Pagination : $56-57$

ISSN : 1150-594X

\section{Référence électronique}

Danielle Vingler, «Lecture critique du spectacle Équations ». Coulisses [En ligne], 14 | Printemps 1996,

mis en ligne le 20 mars 2019, consulté le 25 octobre 2019. URL : http://journals.openedition.org/ coulisses/4715 ; DOI : 10.4000/coulisses.4715

Ce document a été généré automatiquement le 25 octobre 2019

Coulisses 


\title{
Lecture critique du spectacle Équations
}

\author{
Danielle Vingler
}

Création collective du Théâtre Universitaire de Franche-Comté

Animateur-metteur en scène Joseph MELCORE

1 Entre l'apparition, au début du spectacle, des deux enfants clowns qui restituent les représentations des savants que se font les vrais enfants ${ }^{1}$, et celle de ces mêmes enfants clowns qui, à la fin du spectacle, retirent leur déguisement «Tournesol » pour nous donner une conception du monde plus poétique et rassurante: une heure de vrai spectacle.

\section{Le thème}

2 Via une machine accoucheuse, style «Génération Protéus »" remise au goût du jour à travers le jargon et les agitations bio-technologico-mathématico-informatiques de ses assistants, un savant donne naissance à une blonde créature, tout de blanc vêtue, femme, belle, porteuse de tous les espoirs scientifiques de l'humanité, et chérie par ses géniteurs-hommes de science.

3 Mais un rêve brouille cette vision idyllique et couche sur pellicule le négatif des personnages de cette première partie.

4 De blonde immaculée, la créature devient brune ténébreuse, les savants troquent blouses blanches contre combinaisons blafardes, chaussent lunettes, gants et bottes noires pour évoluer dans un univers mystérieux, éclairé des seules torches électriques aux faisceaux lumineux sculpteurs de visages inquiétants.

5 Ils prennent en otage les sublimes découvertes pour les livrer au système marchand jusques et y compris la médecine. Pères nourriciers d'une Publicité des plus folles, mêlant chirurgie esthétique, lessive-lave-plus-blanc et préservatifs en dentelle, ils ensorcellent, sous l'œil bienveillant de Science-Noire, les pauvres consommateurs de rêve que nous sommes. 
6 Pauvre M. Dupont aussi, réduit à sa plus simple expression de squelette, dépecé sous couvert de guérison, au cours d'une cérémonie quasi sacrificielle - juteuse il est vrai digne des peplum qui font encore les délices de nos fantasmes !

Pauvre Nature souillée par la marée noire sortant de la bouche vipérine de ScienceNoire.

Ce spectacle, à travers ce thème et les péripéties qui en découlent, pose le problème du « double visage » de la Science et suggère quelques réflexions.

9 Pas vraiment de manichéisme tranché entre Blonde-Science-Immaculée et ses serviteurs, et Brune-Science-Ténébreuse et ses acolytes. Dans cette affaire il y a, il est vrai, les "Mauvais », ceux qui détournent (économie de marché et amour du profit obligent). Il y a encore ceux qui assouvissent les instincts purificateurs de la «bête immonde »!

10 Mais il y a aussi ceux qui s'exaltent, en quête d'absolu, en jouant aux apprentis sorciers, sans réfléchir vraiment aux conséquences de leurs travaux (d'autres exemples auraient pu être choisis). Sont-ils tellement meilleurs que les autres? La jouissance aveugle de leur découverte et du pouvoir scientifique est-elle un meilleur moteur que celui de la vénalité ? Pourtant, l'attitude affolée de notre savant est en quelque sorte son mea culpa. Quand au sortir de son cauchemar il se rend compte de son impuissance à endiguer les dérives qu'il a involontairement déchaînées, il nous invite à faire une discrimination. Entre ceux des expérimentateurs conscients et inconscients dont il fait partie, nous sommes conduits à préférer malgré tout la deuxième catégorie à la première. Ne fait-il pas preuve de lucidité quand il met dans la bouche de sa créature ces sages paroles : «La Science est quelque chose de trop sérieux pour qu'on la laisse aux seuls scientifiques ».

De mon point de vue, le message passe bien grâce à une écriture forte de symbolisme et d'humour grandguignolesque. Frankenstein et Dr. Folamour pointent l'oreille. Il y a là du Cami et de la Bande Dessinée, mais surtout beaucoup de rigueur dans la mise en place de ce grand jeu, livre d'image. Les acteurs savent exactement ce qu'ils font et pourquoi ils le font. Ils excellent à brouiller les pistes et à faire surgir au moment où on s'y attend le moins des images fortes. Telle cette odalisque de Matisse, toute de formules mathématiques habilement tracées, en une seconde dévoilées. Ou encore ce défilé d'ossements, style équarrissage à jet d'hémoglobine, remplacé dans l'instant par une procession d'une esthétique grandiose, avec grands prêtres portant religieusement d'énormes bocaux remplis de liquides colorés. 


\section{Équations}

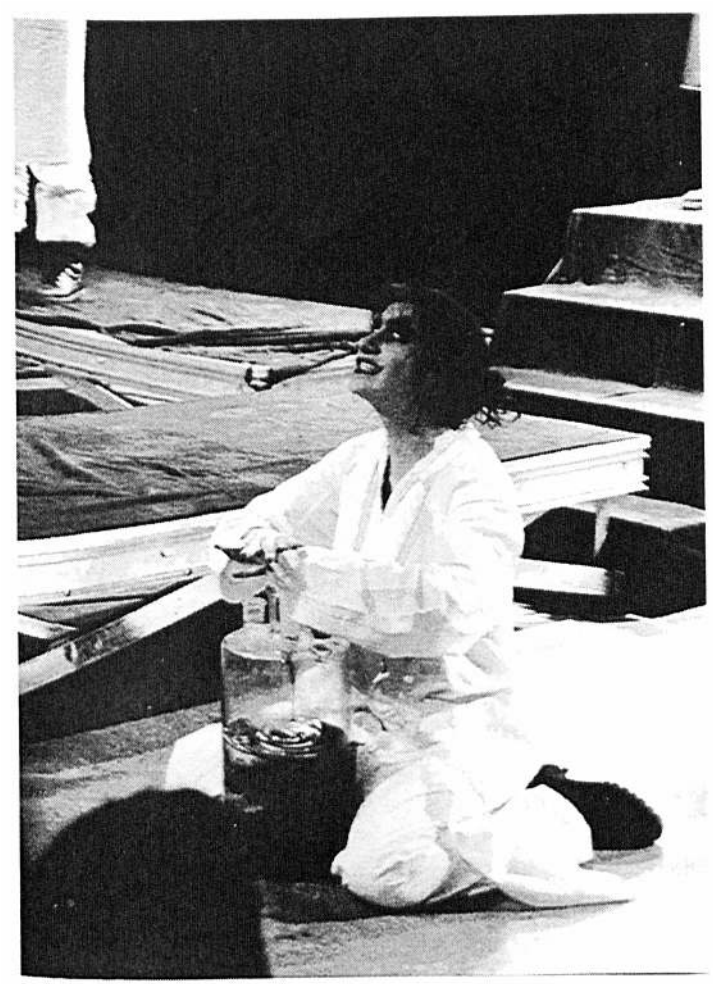

Photo Paola Leitao

12 L'efficacité de ce genre de spectacle repose justement sur la distanciation induite entre la forme et la réalité des contenus.

Si l'on perçoit bien que les jouets de la Pub c'est nous, que le squelette c'est nous, que les flots de marée noire c'est encore nous, il est plus difficile de percevoir l'origine de certains décalages non moins horribles qui sous-tendent d'autres scènes.

Faute de savoir par exemple que l'examen descriptif de quelques spectateurs «cobayes» est tiré des fiches anthropomorphiques mises au point par les médecins nazis pour trier leur population, pour ne pas dire leurs victimes! C'est peut-être cette dimension informative "a posteriori » sur les sources de certains textes qui ont généré le spectacle qui nous manque pour le rattacher totalement à notre réalité. Pourquoi ne pas utiliser par exemple le salut à cette fin?

Information orale ou écrite peu importe, mais information nécessaire à la pleine prise en compte des propos exposés, sans que soit gommé pour autant le propos central lié à la spécificité de l'activité humaine toujours à la recherche d'un monde meilleur, sur un chemin semé de contradictions et qui est bien présent ici.

Une autre qualité de ce spectacle, et non la moindre, est que, pour un spectateur non habitué au Théâtre de Science, il est d'abord plaisir théâtral pur, et joue bien sa fonction d'alerte. Il risquerait toutefois de perdre son impact s'il ne s'inscrivait pas dans un contexte plus large aidant à la vulgarisation scientifique par le théâtre.

Dans cette perspective, il est une excellente introduction au colloque de 1998 qui traitera de cette problématique. 


\section{BIBLIOGRAPHIE}

- La science en poésie. Gallimard

- Lucrèce

- Sophocle : Antigone

- R. de Obaldia, Monsieur Klebs et Rozalie

- M. de Pracontal, L'imposture scientifique en 10 leçons

- J. Carelman, Catalogue des objets introuvables

- Ph. Aziz, Les médecins de la mort

- E. Morin, Science avec conscience

- Articles dans différentes revues scientifiques (Science et Vie, Eurêka, etc.)

- Le Serment d'Hippocrate

\section{ANNEXES}

\section{Diffusion}

- 06 mars 1996 :

Première à Besançon dans le cadre des $7^{\mathrm{e}}$ Rencontres Internationales ThéâtreUniversité

- 23 mars 1996 :

Présentation d'extraits dans le cadre du Salon de la Jeunesse à Besançon

- 05 avril 1996 :

$7^{\mathrm{e}}$ Rencontres Théâtrales de Lyon

- 09 avril 1996 :

$5^{\mathrm{e}}$ Nuits des Équinoxes de Montpellier

- 15 avril 1996 :

Cracovie (Pologne)

- 11 mai 1996 :

Giromagny (Théâtre des deux Sapins)

- 16 mai 1996 :

$8^{\mathrm{e}}$ Biennale de Morteau, Théâtre Municipal

\section{NOTES}

1. « Dessine-moi un savant » (Science et imaginaire, enquête du CAES du CNRS)

2. Film de fiction sur l'ordinateur de Donald Cammel

3. B. Brecht, La résistible ascension d'Arturo Ui 